\title{
Functionally Graded Interfaces: Role and Origin of Internal Electric Field and Modulated Electrical Response
}

\author{
Deepam Maurya, ${ }^{* \dagger}{ }^{\dagger}$ Yuan Zhou, $^{\dagger}$ Bo Chen, ${ }^{\dagger}$ Min-Gyu Kang, ${ }^{\dagger}$ Peter Nguyen, ${ }^{\ddagger}$ Mantu K. Hudait, ${ }^{\ddagger}$ \\ and Shashank Priya* ${ }^{* \dagger}$ \\ ${ }^{\dagger}$ Bio-inspired Materials and Devices Laboratory (BMDL), Center for Energy Harvesting Materials and Systems (CEHMS), Virginia \\ Tech, Blacksburg, Virginia 24061, United States \\ ${ }^{*}$ Bradley Department of Electrical and Computer Engineering, Virginia Tech, Blacksburg, Virginia 24061, United States
}

\section{Supporting Information}

ABSTRACT: We report the tunable electrical response in functionally graded interfaces in lead-free ferroelectric thin films. Multilayer thin film graded heterostructures were synthesized on platinized silicon substrate with oxide layers of varying thickness. Interestingly, the graded heterostructure thin films exhibited shift of the hysteresis loops on electric field and polarization axes depending upon the direction of an applied bias. A diode-like characteristics was observed in currentvoltage behavior under forward and reverse bias. This modulated electrical behavior was attributed to the perturbed dynamics of charge carriers under internal bias (self-bias) generated due to the increased skewness of the potential wells. The cyclic sweeping of voltage further demonstrated memristor-like current-voltage behavior in functionally graded heterostructure devices. The presence of an internal bias assisted the generation of photocurrent by facilitating the separation of photogenerated charges. These novel findings provide opportunity to design new circuit components for the next generation of microelectronic device architectures.

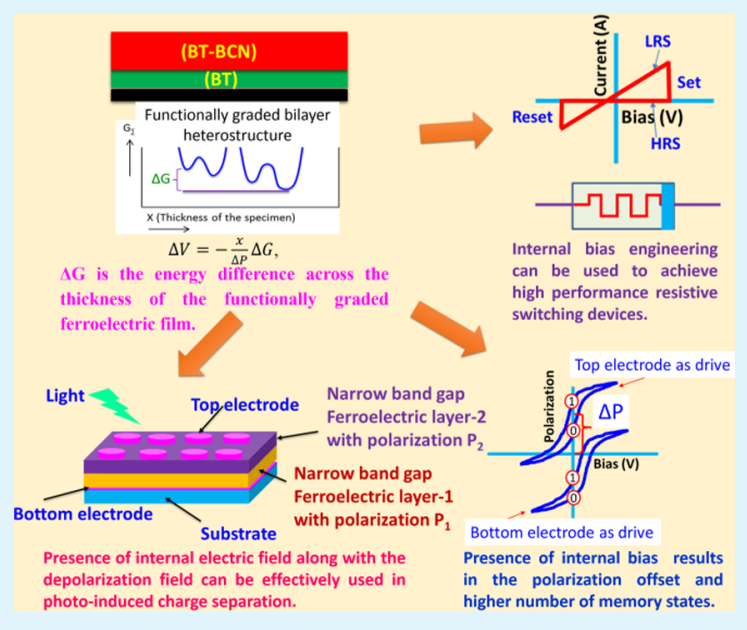

KEYWORDS: functionally graded interfaces, thin film, internal bias, ferroelectric, electrical transport

\section{INTRODUCTION}

Functionally graded ferrolectric thin film heterostructures have attracted significant attention in recent years due to their unusual ferroelectric and dielectric response. These graded ferroelectric systems have been projected for the application as gate dielectric in ferroelectric memory field-effect transistors (FeMFETs), ${ }^{1-4}$ providing a nondestructive readout operation with high density. The stored dipole moments in the ferroelectric material can adjust the threshold voltage of a FeMFET, and thus drain current of each switching state can be discriminated and identified as a logic state in memory. In such applications, the switching capability and energy loss during the operation of ferroelectric gate is quite important. Both of these parameters (switching and energy loss) are dependent upon the polarization-electric field hysteresis behavior of a ferroelectric material. Graded heterostructures provide an opportunity for tuning the performance of these devices. In order to develop ferroelectric systems with adequate hysteretic behavior for circuit and storage components, efforts have been made on fabricating multilayer thin films ${ }^{5-7}$ with internal/built-in field across the interaface due to finite polarization coupling between the layers. Moreover, the electrical transport under built-in electric field and perturbed dynamics of charge carriers can be utilized to achieve resitive switching for memrister applications. 8 This built-in electric field can be instrumental in photoinduced charge separation for achieving the high efficiency optoelectronic components. ${ }^{9,10}$ Furthermore, the built-in electric field in ferroelectric devices results in polarization offset, which can be utilized for high sensitivity infrared detectors.

Attempts have been made to synthesize multilayer thin film structures with built-in bias field using $(\mathrm{BaSr}) \mathrm{TiO}_{3}(\mathrm{BST})$ or $\mathrm{Pb}(\mathrm{ZrTi}) \mathrm{O}_{3}$ (PZT) based material systems. ${ }^{11,12}$ Because lead is hazardous and toxic to the environment, there is a challenge in developing new generation of components based on PZT due to the low volatilization temperature of lead oxide. ${ }^{13-15}$ BSTbased material systems have Curie temperature in the vicinity of room temperature, ${ }^{12}$ which limits the temperature range of operation in a fabricated FeMFET. In literature, researchers have demonstrated variety of multilayer ferroelectric thin film structures, mostly, based upon the PZT and BST systems. ${ }^{16-20}$ Such thin film structures have been appealing for a new class of

Received: July 19, 2015

Accepted: September 17, 2015 
transcapacitive ferroelectric devices, which are viewed to be the dielectric equivalent of the semiconductor junction devices. ${ }^{11,21}$ However, they require a new material system and architecture to overcome the challenges imposed by environment, temperature range, ferroelectric hysteresis and current-voltage behavior. Here, a systematic investigation is presented on new lead-free graded heterostructures, which can overcome above challenges and provide an opportunity to develop practical components.

We have synthesized functionally graded interfaces in the lead-free thin films of $\mathrm{BaTiO}_{3}$ (BT) and $0.975 \mathrm{BaTiO}_{3}-$ $0.025 \mathrm{Ba}\left(\mathrm{Cu}_{1 / 2} \mathrm{Nb}_{2 / 3}\right) \mathrm{O}_{3}(\mathrm{BT}-\mathrm{BCN})$ and successfully demonstrated the modulation of ferroelectric behavior and electrical response in such structures. The design of lead-free piezoelectric/ferroelectric $\mathrm{BT}-\mathrm{BCN}$ with superier functional response has been discussed elsewhere. ${ }^{22}$ Briefly, several compositions were synthesized over a wide compositional range and characterized. The composition $0.975 \mathrm{BaTiO}_{3}-$ $0.025 \mathrm{Ba}\left(\mathrm{Cu}_{1 / 2} \mathrm{Nb}_{2 / 3}\right) \mathrm{O}_{3}$ was found to exhibit superior piezoelectric response with $d_{33} \sim 330 \mathrm{pC} / \mathrm{N}$ and $k_{\mathrm{p}} \sim 46 \%$. ${ }^{22}$ The piezoelectric response of $\mathrm{BT}-\mathrm{BCN}$ was comparable to that of commercial lead-based PZT (Pz23, Ferroperm) with $d_{33}=330$ $\mathrm{pC} / \mathrm{N}$ and $k_{\mathrm{p}}=0.52$. Moreover, $\mathrm{BT}-\mathrm{BCN}$ had a tetragonal crystal structure similar to that of $\mathrm{BaTiO}_{3}$. The differences in tetragonality and polarization of various ferroelectric layers resulted in strain and polarization gradient across the thickness of thin film that induced the translation of the $P-E$ hysteresis loop on E-field and polarization axes, diode-like currentvoltage $(I-V)$ behavior, and asymmetry in capacitance-voltage $(C-V)$ plots. The $J-V$ characteristics $(J$ represents the current density) exhibited high rectification ratio for graded heterostructure. The functionally graded interfaces in the thin film architectures further exhibited memristor-like resistive switching behavior. Interestingly, the photovoltaic effect was observed in the specimen having functionally graded interfaces with internal electric field. Here, we provide a fundamental understanding of the modulated electrical response and the role of internal electric fields in imparting these desired characteristics to the functionally graded ferroelectric heterostructures.

\section{EXPERIMENTAL SECTION}

2.1. Thin Film Deposition. The lead-free stoichiometric $\mathrm{BaTiO}_{3}$ and $0.975 \mathrm{BaTiO}_{3}-0.025 \mathrm{Ba}\left(\mathrm{Cu}_{1 / 3} \mathrm{Nb}_{2 / 3}\right) \mathrm{O}_{3}$ targets were synthesized using conventional mixed oxide processing route. The detailed synthesis process for $\mathrm{BT}$ and $\mathrm{BT}-\mathrm{BCN}$ can be found elsewhere. ${ }^{23}$ The single layer, bilayer, and trilayer functionally graded thin film heterostructures were deposited on $\mathrm{Pt}(111) / \mathrm{Ti} / \mathrm{SiO}_{2} / \mathrm{Si}$ substrate (Inostek, Seoul, Korea) using a vacuum chamber with $\operatorname{KrF}(\lambda=248$ $\mathrm{nm}$ ) excimer pulsed laser deposition (PLD) system at energy density of $\sim 2.5 \mathrm{~J} / \mathrm{cm}^{2}$ with a laser repetition rate of $10 \mathrm{~Hz}$. The deposition was performed in an oxygen pressure of 100 mTorr with substrate temperature of $\sim 800{ }^{\circ} \mathrm{C}$. All the films were deposited for 20000 pulses with typical thickness of 250-280 nm under similar deposition conditions. The only varying parameter was the film thickness ratio between $\mathrm{BT}$ and $\mathrm{BT}-\mathrm{BCN}$. The variation in the $\mathrm{BT}$ and $\mathrm{BT}-\mathrm{BCN}$ layers was achieved by controlling the repetition rate of the laser pulses. We used Philips X'pert Pro X-ray diffractometer (Almelo, The Netherlands) for recording XRD patterns at room temperature. All the samples were found to crystallize in $\mathrm{ABO}_{3}$ type perovskite structure with tetragonal symmetry, as shown in Figure S1 (Supporting Information). These films also exhibit preferred crystallographic orienation along $\langle 111\rangle$, as shown in Figure S1.

2.2. Morphology and Microstructure. The morphology of each thin film was observed by field emission scanning electron microscopy
(SEM), as shown in Figure S2 (Supporting Information). The morphology and microstructure of the bilayer thin film was investigated using transmission electron microscopy (TEM). For fabricating electron transparent TEM sample, we used standard grinding and ion-milling methods. The TEM images were recorded using a FEI Titan 300 microscope. A scanning probe microscope (Bruker Dimension Icon, Billerica, MA) coupled with conductive platinum coated silicon cantilever was used to perform the piezoresponse force microscopy (PFM) on the single layer and bilayer thin films. The PFM images were recorded at frequency of $1 \mathrm{MHz}$ under applied AC voltage of $3 \mathrm{~V}$. The morphology images were also recorded concomitantly with the PFM images.

2.3. Ferroelectric and Electrical Characterization. For electrical measurements, the top platinum $(\mathrm{Pt})$ electrode with diameter of $500 \mu \mathrm{m}$ was deposited by rf-sputtering via a shadow mask at room temperature. Both the ferroelectric $P-E$ hysteresis loops and the $I-V$ characteristics were recorded using a ferroelectric tester (Radiant technology). A Keithley 4200-SCS Parameter Analyzer connected with Cascade Summit 9000 Probe Station was used for cyclic current - voltage measurements. The $C-V$ measurements were performed using a HP 4194A impedance analyzer at $500 \mathrm{mV}$ AC voltage and frequency of $1 \mathrm{kHz}$.

2.4. Photovoltaic Measurements. The photovoltaic performance of the thin films was analyzed under one sun AM $1.5(100 \mathrm{~mW} /$ $\mathrm{cm}^{2}$ ) illumination with a solar simulator (150 W Sol 2ATM, Oriel). The power output of the lamp was calibrated to 1 Sun (AM1.5G, 100 $\mathrm{mW} / \mathrm{cm}^{2}$ ) using a certified Si reference cell. During the photovoltaic measurements, the current-voltage characteristics were recorded with a Keithley digital source meter (model 2400).

\section{RESULTS AND DISCUSSION}

\subsection{Design of Various Thin Film Architectures and} Microstructural Analysis. The schematic representations of various graded thin film heterostructures, studied in this work, are depicted in Figure 1a. The top view and the cross-sectional SEM image of the bilayer $0.5(\mathrm{BT})-0.5(\mathrm{BT}-\mathrm{BCN})$ structures are shown in Figure 1b,c. The cross-sectional TEM micrograph of functionally graded bilayer heterostructure thin film is shown in Figure 2a, and the inset shows the magnified view of the
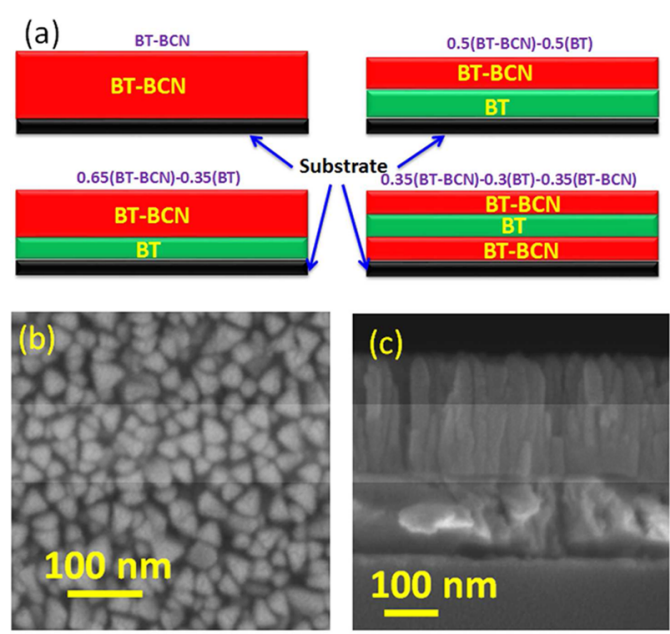

Figure 1. Thin film device architectures and morphology. (a) Schematic representation of various graded thin film heterostructures, (b) SEM micrographs of bilayer thin film with equal thickness of BT and $\mathrm{BT}-\mathrm{BCN}$ (top view), and (c) cross-sectional view of the structure shown in panel $b$. Schematic of polarization electric field $(P-E)$ loops for (e) bulk and (f) thin film structures and (insets) corresponding trilayer functionally graded heterostructures. Note the translated $P-E$ loop for graded thin film trilayer structure. 

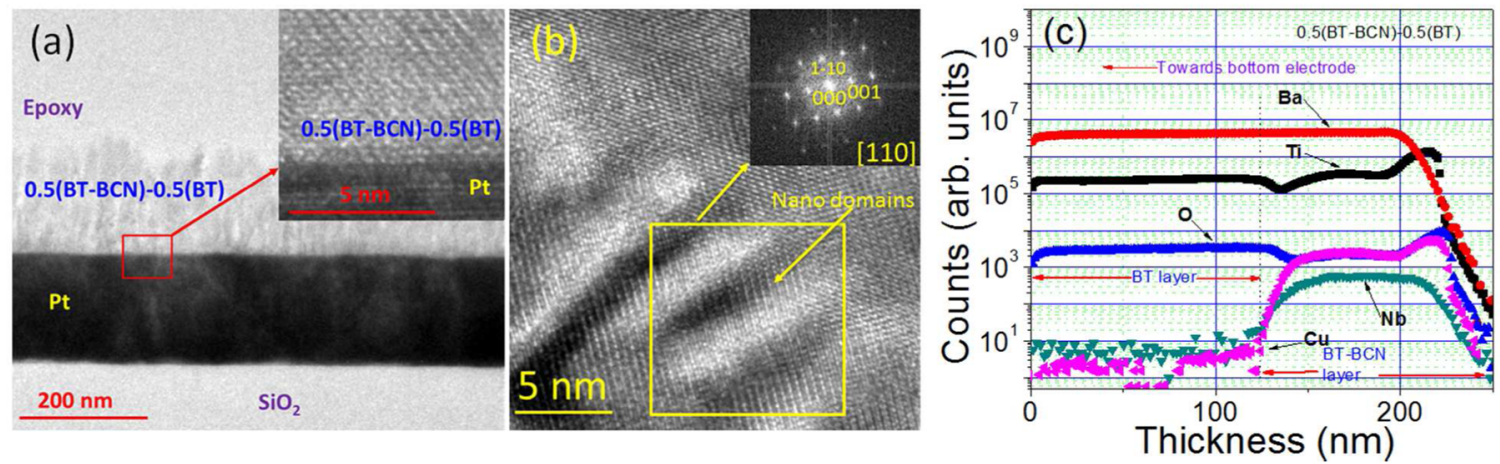

Figure 2. Interface and compositional analysis using TEM and SIMS, respectively. (a) Bright field TEM image of the bilayer 0.5(BT-BCN)0.5(BT) thin film cross section and (inset) HR-TEM image of lattice fringes across the interfacial region. (b) HR-TEM image of the thin film showing stripe-like ferroelectric nanodomains from [110] direction and (inset) FFT pattern corresponding to the rectangular region. (c) Time-offlight secondary-ion mass spectra for the $0.5(\mathrm{BT}-\mathrm{BCN})-0.5(\mathrm{BT})$ bilayer thin film. Note the variation of various elements across the thickness of the film. Presence of $\mathrm{Cu}$ and $\mathrm{Nb}$ suggests bilayer structure of the thin film.

interface without defects. The cross-sectional TEM and SEM micrographs undoubtedly indicate the columnar growth of the bilayer thin film. The selected area electron diffraction (SAED) from the cross-section of the film shows evidence of the preferred orientation in $\langle 111\rangle$ direction of the deposited thin film, as shown in Supporting Information (Figure S3). This is in line with the $\langle 111\rangle$ preferred orientation observed in the XRD-patterns of various films (Figure S1). The indexing of the fast Fourier transform (FFT) pattern corresponding to the rectangular area marked in the image, suggested [110] zone axis. Figure $2 b$ shows a HR-TEM lattice fringe image with stripe like nanodomains. The smaller size nanodomains have been found to switch with ease as compared to their macroscopic counterpart due to higher domain mobility. ${ }^{24,25}$ The high density of the ferroelectric domains results in enhanced domain wall mobility, which in turn, gives rise to enhanced functional response. However, in polycrystalline bulk systems, the domain size decreases with the decrease in the grain size until a critical size is reached, beyond which, domain walls are clamped by the geometrical restrictions imposed through the grain boundaries. Due to very small differences in compositional variation between the $\mathrm{BT}$ and $\mathrm{BT}-\mathrm{BCN}$ layer (BT-BCN contains $\mathrm{Cu}=0.8$ atomic $\%$ and $\mathrm{Nb}=$ atomic $1.6 \%)$, the bilayer structure was not clearly observed in the TEM. Moreover, due to small concentration of $\mathrm{Nb}$ and $\mathrm{Cu}$ atoms, energy dispersive spectroscopy (EDS) was not able to detect these elements. Therefore, to investigate distribution of $\mathrm{Cu}$ and $\mathrm{Nb}$ across the thickness, we performed time-of-flight secondary ion mass spectrometry (TOF-SIMS) analysis on bilayer $0.5(\mathrm{BT}-\mathrm{BCN})-0.5 \mathrm{BT}$ thin film, as shown in Figure 2c. For SIMS depth profiling, the Cameca IMS 7f GEO magnetic sector secondary ion mass spectrometer was used. The crater map created after recording the SIMS spectra is provided in the Supporting Information (Figure S4). The SIMS depth profiling results indicated that $\mathrm{Ba}, \mathrm{Ti}$, and $\mathrm{O}$ elements were uniformly present across the thickness. However, $\mathrm{Cu}$ and $\mathrm{Nb}$ was present only across the half thickness $(\sim 125 \mathrm{~nm})$ of the film. This confirmed the presence of composition gradient in 0.5(BTBCN) $-0.5 B T$ bilayer thin film. In SIMS results, the elemental concentration was not quantified due to lack of the standard specimen for the calibration.

The bulk functional properties ${ }^{23,26}$ of BT and BT-BCN materials previously reported are summarized in Table 1 . One can find a significant difference in the functional and structural
Table 1. Bulk Functional Properties of the Materials ${ }^{23,26}$ Deposited As Thin Film Structures

\begin{tabular}{lccccc} 
& \multicolumn{5}{c}{ bulk properties } \\
\cline { 2 - 6 } sample & $\begin{array}{c}d_{33} \\
(\mathrm{pC} / \mathrm{N})\end{array}$ & $\varepsilon_{33}^{T} / \varepsilon_{o}$ & $\begin{array}{c}P_{\mathrm{r}} \\
\left(\mu \mathrm{C} / \mathrm{cm}^{2}\right)\end{array}$ & $\begin{array}{c}\text { tetragonality } \\
(\mathrm{c} / \mathrm{a})\end{array}$ & $\begin{array}{c}T_{\mathrm{c}} \\
(\mathrm{K})\end{array}$ \\
$\mathrm{BT}$ & 144 & 2100 & 17 & 1.010 & 398 \\
$\mathrm{BT}-\mathrm{BCN}$ & 330 & 3000 & 5 & 1.004 & 353 \\
\hline
\end{tabular}

properties of these two materials. These differences lead to different structural and electrical response in the thin film form. The tetragonality (c/a) was calculated using (200) and (002) $\mathrm{X}$-ray diffraction peaks and was found to be 1.01 and 1.006 for $\mathrm{BT}$ and $\mathrm{BT}-\mathrm{BCN}$ powders, respectively. ${ }^{23}$ This difference in tetragonality between $\mathrm{BT}$ and $\mathrm{BT}-\mathrm{BCN}$ defined the difference in principle strain $S_{s}$, given as $S_{s}=(c / a-1)$. The magnitude of $S_{\mathrm{s}}$ was found to be 0.01 for BT and 0.006 for BT-BCN, which could result in the strain gradient across the interface between the two coupled layers. The large difference in remnant polarization created a functionally graded structure across the thickness of the film.

3.2. Effect of Functional Grading on Ferroelectric Response. Figures $3 a$ and $4 a$ show the surface morphology for
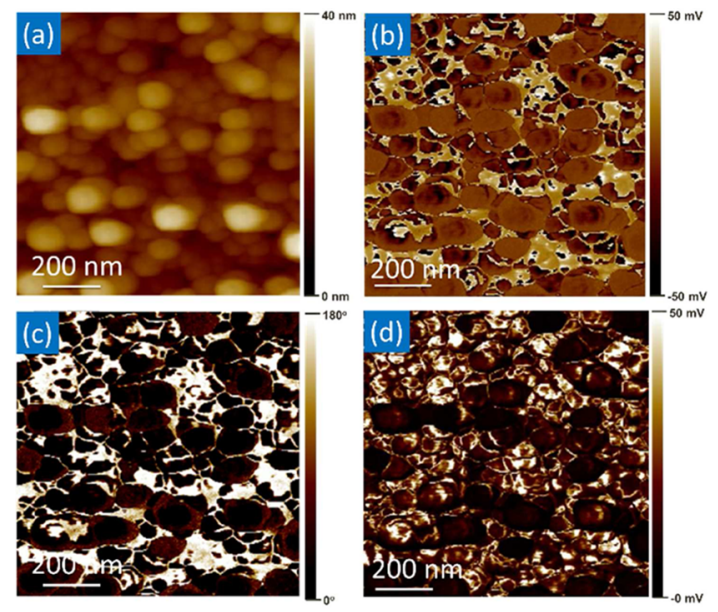

Figure 3. I PFM to study local piezoelectric response in single-layer $\mathrm{BT}-\mathrm{BCN}$ thin film. Recorded simultaneously (a) surface topography, (b) PFM piezoresponse image, (c) PFM phase image showing ferroelectric domains, and (d) PFM amplitude image. 

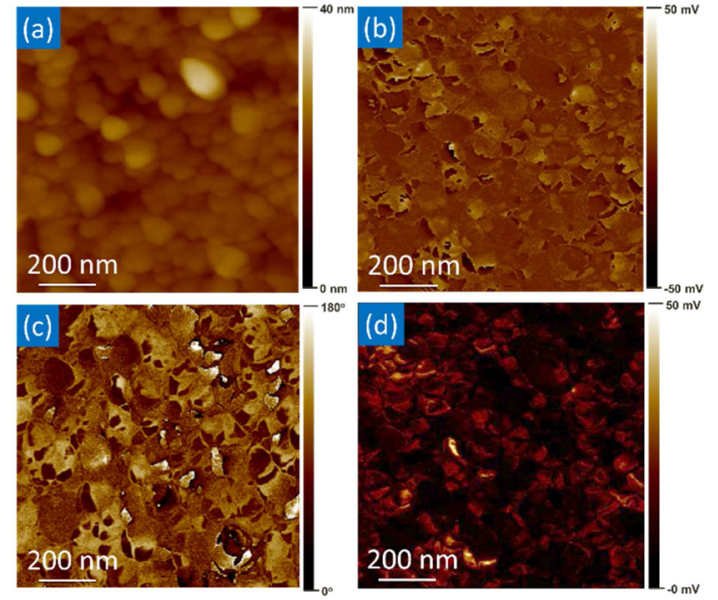

Figure 4. I PFM to study local piezoelectric response in bilayer $0.65(\mathrm{BT}-\mathrm{BCN})-0.35(\mathrm{BT})$ thin film. Recorded simultaneously (a) surface topography, (b) PFM piezoresponse image, (c) PFM phase image showing ferroelectric domains, and (d) PFM amplitude image.

single layer $\mathrm{BT}-\mathrm{BCN}$ and $0.65(\mathrm{BT}-\mathrm{BCN})-0.35 \mathrm{BT}$ bilayer thin film, respectively. The piezoresponse, phase and amplitude PFM images are depicted in Figure $3 b-d$ and Figure $4 b-d$ for single and bilayer thin films, respectively. The morphology of both the samples was found to be similar. However, there was a large differences in the piezoelectric response, phase, and amplitude of both the films. The single-layer BT-BCN film showed high contrast in the piezoresponse, phase, and amplitude image. On the other hand, the bilayer thin film exhibited lesser contrast in PFM image, suggesting smaller piezoelectric response. It can be clearly observed that the ferroelectric domains of the bilayer thin film are relatively poled, whereas the single layer film exhibits entirely random domain distribution, as shown in both the phase images (Figures $3 \mathrm{c}$ and $4 \mathrm{c}$ ). This indicates the polarization of the bilayer thin film is probably naturally oriented without external bias. We measured the local piezoresponse hysteresis using PFM for the single layer BT-BCN and bilayer 0.65(BT$\mathrm{BCN})-0.35 \mathrm{BT}$ thin films, which indicated polarization switching in these films at the nanoscale, as shown in Figure S5 (Supporting Information). We believe that the presence of internal bias played a major role in the origin of the difference in the functional response, measured, using PFM. ${ }^{27}$ The presence of an internal bias has been previously found to substantially modulate the domain morphology. ${ }^{28}$

Figure 5 shows the $P-E$ hysteresis recorded using bipolar signal at $2 \mathrm{kHz}$ for various thin films having functionally graded heterostructures. For this, the polarization hysteresis loops at different frequencies and fields (not presented here) were measured for optimization of the measurement parameters. Also, contribution of the leakage current has been found to be lower at higher frequency. ${ }^{29}$ To perform a comparative analysis for different architectures, we selected the $P-E$ loops measured at a bipolar signal with a frequency of $2 \mathrm{kHz}$. From these figures, we can clearly observe the translation of $P-E$ hysteresis loop on the $E$ field and polarization axes. The shift along the polarization axis could be attributed to the internal bias present at the interface. This measurement was performed with drive and return probe on top and bottom electrode, respectively. Interestingly, on reversing the polarity of the hysteresis measurements (i.e., bottom electrode was used as a drive electrode and top electrode was used as a return), the $P-E$ loop was shifted in opposite direction as depicted in the insets of Figure 5 for different systems. The magnitude of translation of $P-E$ loop was found to be same under both types of measurement configurations. Therefore, the direction of translation of the hysteresis loops was found to be dependent
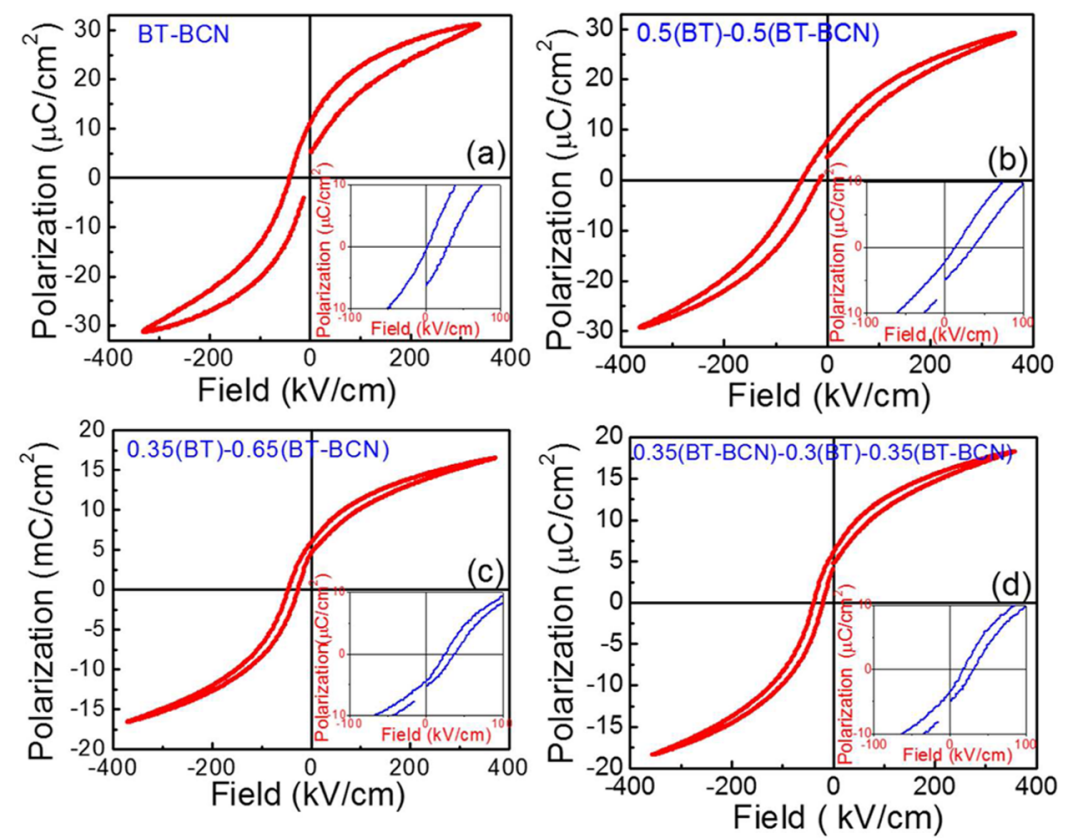

Figure 5. I Modulated ferroelectric behavior in functionally graded thin film architectures. Translation of $P-E$ hysteresis on polarization and $E$-field axes for (a) BT-BCN single layer, (b) 0.5(BT) $-0.5(\mathrm{BT}-\mathrm{BCN}$ ) bilayer having equal thickness, (c) 0.35(BT) $-0.65(\mathrm{BT}-\mathrm{BCN})$ bilayer having unequal thickness, and (d) $0.35(\mathrm{BT}-\mathrm{BCN})-0.30(\mathrm{BT})-0.35(\mathrm{BT}-\mathrm{BCN})$ trilayer functionally graded heterostructure thin films. Insets are showing $P-E$ loops measured under similar conditions but with bottom electrode as drive and top electrode as return. On interchanging electrode (i.e., bottom electrode as drive and top electrode as return), shift of hysteresis in opposite direction was noticed. 

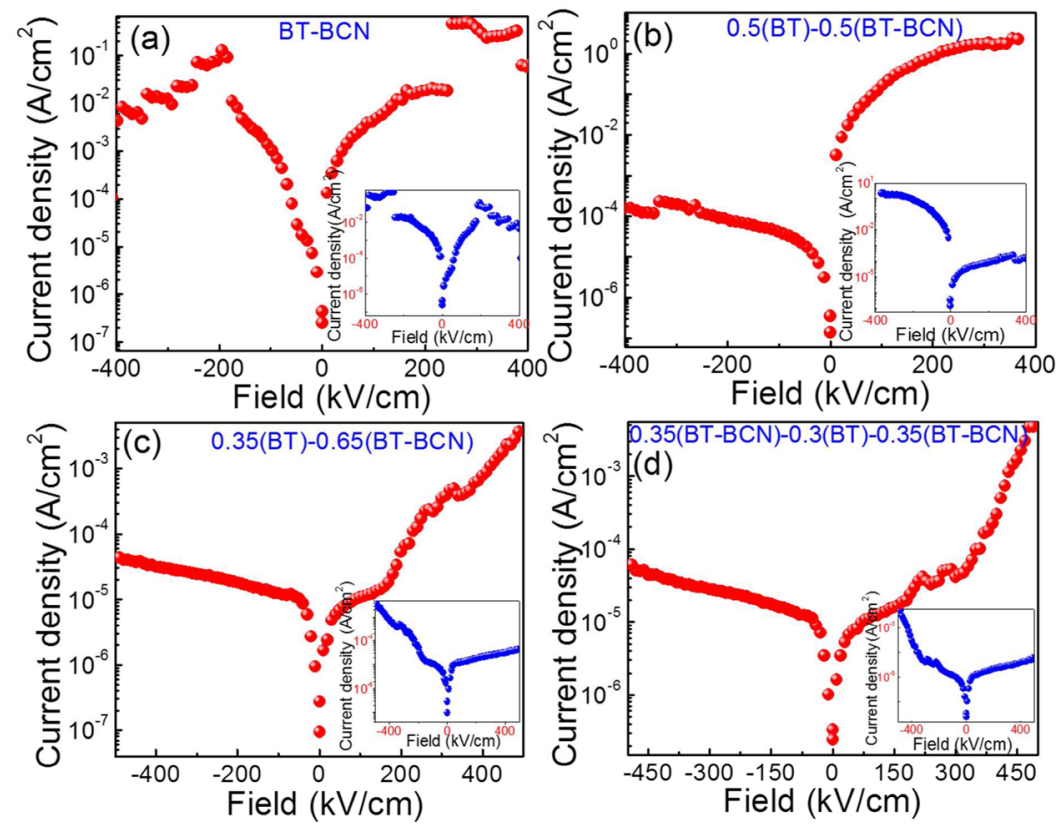

Figure 6. I Current-voltage characteristics with high rectification behavior in functionally graded thin film heterostructures devices. Current density $(J)$ versus $E$-field plots for (a) BT-BCN single layer, (b) 0.5(BT) $-0.5(\mathrm{BT}-\mathrm{BCN})$ bilayer with equal thickness, (c) $0.35(\mathrm{BT})-0.65(\mathrm{BT}-\mathrm{BCN})$ bilayer with unequal thickness, and (d) $0.35(\mathrm{BT}-\mathrm{BCN})-0.30(\mathrm{BT})-0.35(\mathrm{BT}-\mathrm{BCN})$ trilayer functionally graded thin film heterostructures. (Insets) Current density versus $E$-field plots measured under similar conditions but with bottom electrode as drive and top electrode as return.
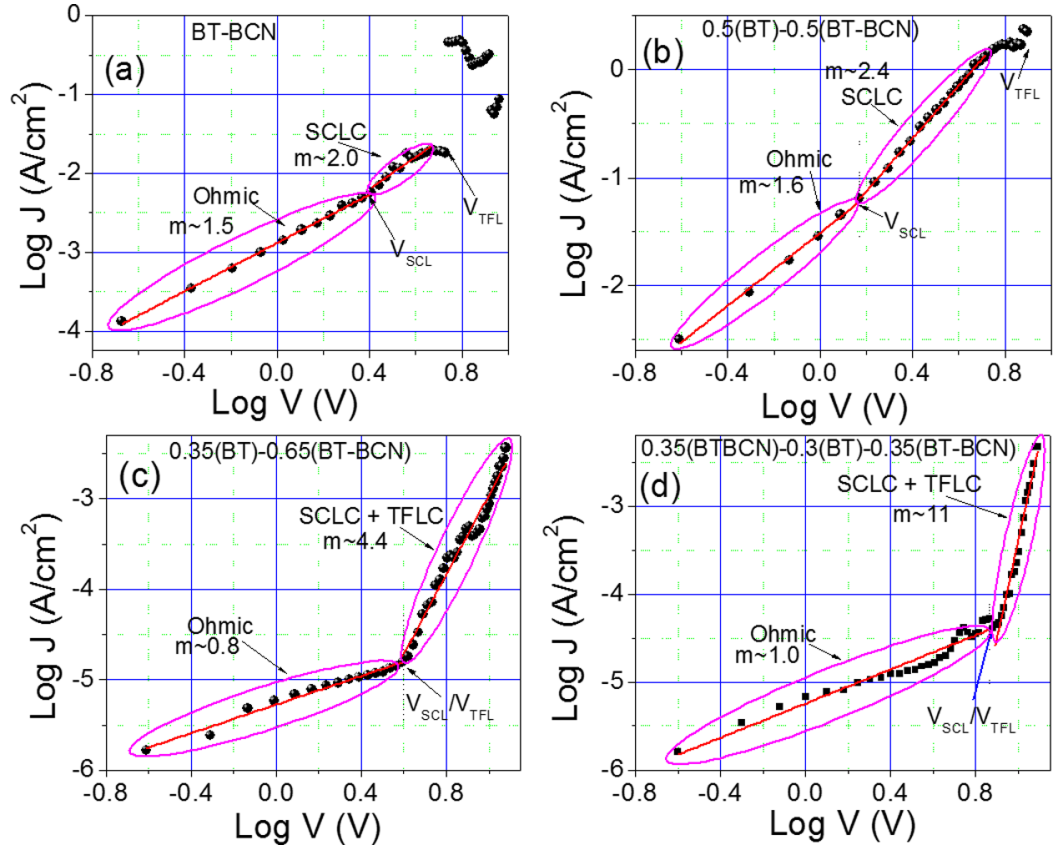

Figure 7. I Log-Log plots of current-voltage characteristics in forward bias showing different mechanisms of electrical transport. Log $(J)$ versus Log $(V)$ plots for (a) BT-BCN single layer, (b) 0.5(BT) $-0.5(\mathrm{BT}-\mathrm{BCN})$ bilayer with equal thickness, (c) $0.35(\mathrm{BT})-0.65(\mathrm{BT}-\mathrm{BCN})$ bilayer with unequal thickness, and $(\mathrm{d}) 0.35(\mathrm{BT}-\mathrm{BCN})-0.30(\mathrm{BT})-0.35(\mathrm{BT}-\mathrm{BCN})$ trilayer functionally graded thin film heterostructures. On the basis of the slope $(m)$ obtained from linear fitting, regions having different slopes are marked on various figures.

on the applied bias (forward or reverse) confirming the presence of a strong internal $E$-field. The centers of the $P-E$ loops intersecting the $x$-axes were at $-20,-29,-37$, and -30 $\mathrm{kV} / \mathrm{cm}$ instead of $0 \mathrm{kV} / \mathrm{cm}$ for the BT-BCN, $0.5(\mathrm{BT}-\mathrm{BCN})-$ $0.5 \mathrm{BT}, 0.65(\mathrm{BT}-\mathrm{BCN})-0.35 \mathrm{BT}$, and $0.35(\mathrm{BT}-\mathrm{BCN})-$ $0.3(\mathrm{BT})-0.35(\mathrm{BT}-\mathrm{BCN})$ thin films, respectively. This clearly suggests presence of enhanced internal bias in the functionally graded thin film heterostructures. In a ferroelectric junction, the polarization is expected to influence the charge transport as a result of modified band alignments and carrier concentration depending on the direction of the ferroelectric polarization state. ${ }^{30-32}$ To further understand the effect of functionally graded heterostructures on the electrical response, we performed current-voltage measurements.

3.3. Rectifying Characteristics, Memristor Behavior and Photovoltaic Effect in Functionally Graded Archi- 
tectures. Figure 6 shows the current density versus electric field $(J-E)$ plots for various thin film structures. From these figures, we can clearly notice that the current density is higher in forward bias than that in the reverse bias, exhibiting diodelike characteristics. This also indicates the existence of strong internal bias across the interface. The electrodes were similar in all the thin film samples, which makes it easier to understand the effect of functional grading on the electrical output. Because there was no significant difference between the forward and reverse bias current in single layer $\mathrm{BT}-\mathrm{BCN}$ thin film (Figure $6 \mathrm{a})$, the electrode effect can be assumed to be trivial. To understand the mechanism of conduction, Log $(J)$ versus Log $(V)$ (forward bias) curves were plotted for different thin films structures, as shown in Figure 7. The space charge limited current (SCLC) can be written as ${ }^{33} J=\left(9 \mu \varepsilon_{s} \varepsilon_{0} \theta / 8 d\right) E^{2}$ with $\theta$ $=n_{\mathrm{f}} / n_{t}=\left(N_{c} / N_{t}\right) \exp \left(E_{t}-E_{c} / k T\right)$, where $\theta$ is the ratio of the total density of free electrons $n_{\mathrm{f}}$ and trapped electrons $n_{\mathrm{v}} \mu$ is the mobility of the charge carriers, $\varepsilon_{\mathrm{s}}$ is the static permittivity of thin film, $\varepsilon_{0}$ is the permittivity of free spaces, $d$ is the thickness of film, $N_{\mathrm{c}}$ is the effective density of states in the conduction band, $N_{\mathrm{t}}$ is the trapped electron density, $E_{\mathrm{t}}$ is the energy level of the traps, and $E_{\mathrm{c}}$ is the bottom energy level of the conduction band. The voltage at which rapid increase in the current density occurs is termed as the trap filled limit (TFL), $V_{\mathrm{TFL}}=e \mathrm{~N}_{\mathrm{t}} \mathrm{d} /$ $2 \varepsilon_{s} \varepsilon_{0}$. The $V_{\mathrm{TFL}}$ for various thin films is marked in Figure 7 . The variation in the value of $V_{\mathrm{TFL}}$ suggests varying height of the potential barrier for different thin film architectures. With the increase in an applied bias, electrons were injected into the film. This resulted in higher density of these electrons as compared to the thermally stimulated electrons. An increase in leakage current generally occurs due to the interaction between the positively charged traps and electrons under SCLC conduction. During SCLC regime, charge carriers get trapped and generate a push-back voltage until all traps are filled (trap filled limit). In other words, after capturing large number of electrons, in order to maintain charge neutrality, the charged interfacial region is pushed back (generating push-back voltage) to uncover required amount of positively charge carriers. ${ }^{34}$ At $V_{\mathrm{TFL}}$, the system overcomes push-back voltage and current rises rapidly. ${ }^{35}$ The slope of $\log (J)-\log (V)$ plots can be helpful in delineating the mechanism of electrical condition. On the basis of linear fitting, the $\log (J)-\log (V)$ curves were divided in to different regions as marked in Figure 7. The single-layer BT-BCN thin film was found to have slopes $m \sim 1.5$ and 2.0 suggesting ohmic and space charge limited current (SCLC) conduction mechanism, respectively. The $V_{\mathrm{SCL}}$ is the voltage at which conduction mechanism changes from ohmic to SCL. On the basis of slopes values, bilayer and trilayer thin films were found to exhibit different mechanism of conduction (i.e., ohmic, SCL, and TFL). The drop in current density for BT-BCN single layer thin film may be attributed to the newly created traps. However, the slope of higher field regime was increasingly higher for bilayer thin films with equal $(m \sim 2.4)$ and unequal thickness $(m \sim 4.4)$, and trilayer thin film $(m \sim 11)$. The higher slope could be attributed to the increased internal bias and the nature of the distribution of traps across the thickness in the specimen. ${ }^{36,37}$ However, the mechanism of conduction in highvoltage regime of the unequal bilayer and trilayer thin films may not be purely SCLC, but a cooperative electrical conduction mechanism incorporating SCLC and TFL. In low-voltage regime, the nonlinearity can be attributed to the presence of the point defects (e.g., oxygen vacancies; see next section on defect chemistry) and defect dipoles as a result of $\mathrm{Cu}$ substitution in
BT-BCN. These oxygen vacancies can significantly contribute to the origin of space charges in the ferroelectric materials. ${ }^{38}$

The ratio $1.5 \times 10^{4}$ of the maximum current in forward bias to that in reverse bias, in Figure $6 b$, indicates rectifying current-voltage characteristics of bilayer with equal thickness of BT and BT-BCN layers. This value of rectifying ratio is 2 orders of magnitude higher than those reported for other perovskite based oxides and comparable to that for binary oxide $p-n$ junctions. ${ }^{1}$ The other structures also show the rectifying characteristics, but the ratio is lower than that of bilayer with equal thickness of $\mathrm{BT}$ and $\mathrm{BT}-\mathrm{BCN}$. These $I-V$ measurements were performed by sweeping voltage from zero toward positive and negative directions. However, on cyclic sweeping of the voltage (i.e., sweeping voltage from negative to positive and positive to negative), hysteresis in $I-V$ plots was obtained (Figure 8). These results are representative and recorded after

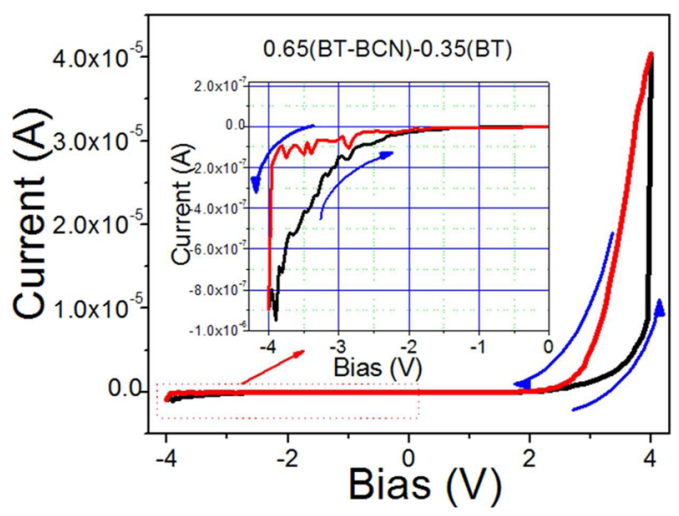

Figure 8. I Memristor-like behavior in functionally graded film heterostructures. Current versus voltage plots under cyclic sweeping indicating memristor like hysteresis for a bilayer $0.35(\mathrm{BT})-0.65(\mathrm{BT}-$ $\mathrm{BCN}$ ) thin film architecture. (Inset) Magnified view from the reverse biased region.

cycling five times. The trapping and detrapping of charge carriers can substantially contribute to the hysteretic behavior of $I-V$ plots. ${ }^{39}$ This memristor-like behavior ${ }^{40}$ can be attributed to the built-in E-field and the trap controlled current conduction mechanism. The rectification behavior reveals that the transport is remarkably influenced by the presence of builtin field across the interface. During forward bias, the device goes through the set operation (high resistance state (HRS) to low resistance state (LRS)). Under increasing reverse bias, the device resets to a previous state. Therefore, the migration of charge carriers under the influence of the internal bias across the interfaces appears to play a vital role in the electrical transport. Especially, oxygen vacancies (being highly mobile $^{38,41}$ and having smaller activation energy $\sim 0.5 \mathrm{eV}^{41}$ ) have been found to play an important role in hysteretic currentvoltage behavior. ${ }^{4142}$ The 0.8 atom $\%$ substitution of $\mathrm{Cu}^{2+}$ (in present case) on $\mathrm{Ti}^{4+}$-site generates equivalent amount of the oxygen vacancies in the system, as discussed later. Moreover, switching of ferroelectric domains can also modulate the internal field across the interface, and thereby, influence the current voltage characteristics. ${ }^{39}$

The presence of internal $E$-field could be very useful in solar energy harvesting, as the sample would not require poling (depolarization field) and the effective charge carrier separation may occur under an internal bias. In order to demonstrate the presence of the internal electric field, photovoltaic measure- 
ments were performed on a bilayer thin film, as shown in Figure 9. The photovoltaic measurements revealed open-circuit

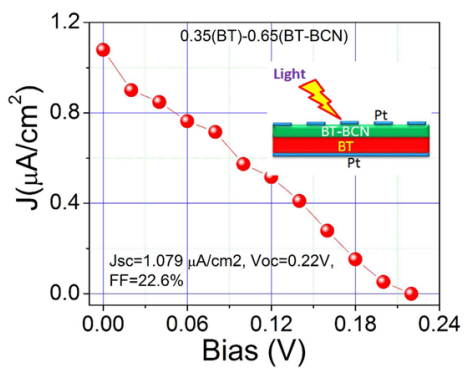

Figure 9. I Built-in bias assisted photovoltaic effect in functionally graded architecture. Current density versus voltage plots under photo illumination for a bilayer $0.35(\mathrm{BT})-0.65(\mathrm{BT}-\mathrm{BCN})$ thin film architecture.

voltage $\left(V_{\text {oc }}\right)$ and short-circuit current $\left(J_{\mathrm{sc}}\right)$ to be $1.079 \mu \mathrm{A} / \mathrm{cm}^{2}$ and $0.22 \mathrm{~V}$, respectively. The photovoltaic measurements on bilayer thin film further suggested fill factor (FF) of $\sim 22.6 \%$ and efficiency of $\eta=0.54 \times 10^{-4} \%$. Even though the $\eta$ is quite low, the photovoltaic result on bilayer thin film clearly demonstrate the presence of internal electric fields. These thin films had high resistivity which reduced the photocurrent. It is worthwhile to mention that we did not find photovoltaic effect in single layer BT-BCN thin film. The small value of various parameters obtained from the photovoltaic measurements can be attributed to the wide optical band gap of BTBCN $(4.38 \mathrm{eV})^{15}$ and BT $(3.5 \mathrm{eV}) .^{43}$ Materials with such large band gaps can only absorb UV radiation, which accounts for only $4 \%$ of the solar radiation. However, such thin film structures, fabricated with narrow band gap $(<3.0 \mathrm{eV})$ ferroelectric materials, may have significantly high photovoltaic performance.

Figure 10 shows the $C-V$ plots for various thin film structures at $1 \mathrm{kHz}$. The difference in the height of the peaks further suggests the presence of the internal bias and finite coupling across the layers. On interchanging the electrodes (top and bottom), the height of the peaks in $C-V$ plots was interchanged, indicating the effect of functional grading. The tunability $\left(=\left(\left(C_{\max }-C_{\min }\right) /\left(C_{\max }\right)\right) \times 100\right)$ calculated from $C-V$ plots of single layer $\mathrm{BT}-\mathrm{BCN}$ thin film capacitor was found to be $\sim 89 \%$. However, the tunability of other graded heterostructure thin film capacitors was found to be 92\% stressing their technological relevance. The high tunability in the functionally graded thin films can be attributed to the enhanced nonlinearity due to built-in bias. ${ }^{28}$

3.4. Origin of Point Defects/Defect Dipoles and Builtin Electric Field. To further explain the modulated electrical response, it is important to understand the defect chemistry in these thin films. According to the chemical formulation of BT$\mathrm{BCN}$, the overall charge neutrality should be maintained by $\mathrm{Cu}^{2+}$ and $\mathrm{Nb}^{5+}$ doping on the Ti-sites. For electroneutrality, the acceptor doping of $\mathrm{Cu}^{2+}$ on the $\mathrm{Ti}^{4+}$-sites gives rise to an equivalent number of oxygen vacancies $\mathrm{V}_{\mathrm{O}}^{\cdot \bullet}$ (Kröger-Vink notation) given as

$$
\mathrm{BaO}+\mathrm{CuO} \rightleftharpoons \mathrm{Ba}_{\mathrm{Ba}}^{\times}+\mathrm{Cu}_{\mathrm{Ti}}^{\prime \prime}+\mathrm{V}_{\mathrm{O}}^{\bullet \bullet}+2 \mathrm{O}_{\mathrm{O}}^{\times}
$$

The oxygen vacancies $\mathrm{V}_{\mathrm{O}}^{\bullet}$ impact the domain wall mobility. The $\mathrm{Cu}_{\mathrm{Ti}}^{\prime \prime}$ vacancies are immobile contrary to the mobile $\mathrm{V}_{\mathrm{O}}^{\bullet \bullet}$ at ambient temperature. ${ }^{44}$ The substitution of $\mathrm{Nb}^{5+}$ occurs on the $\mathrm{Ti}^{4+}$ sites given as
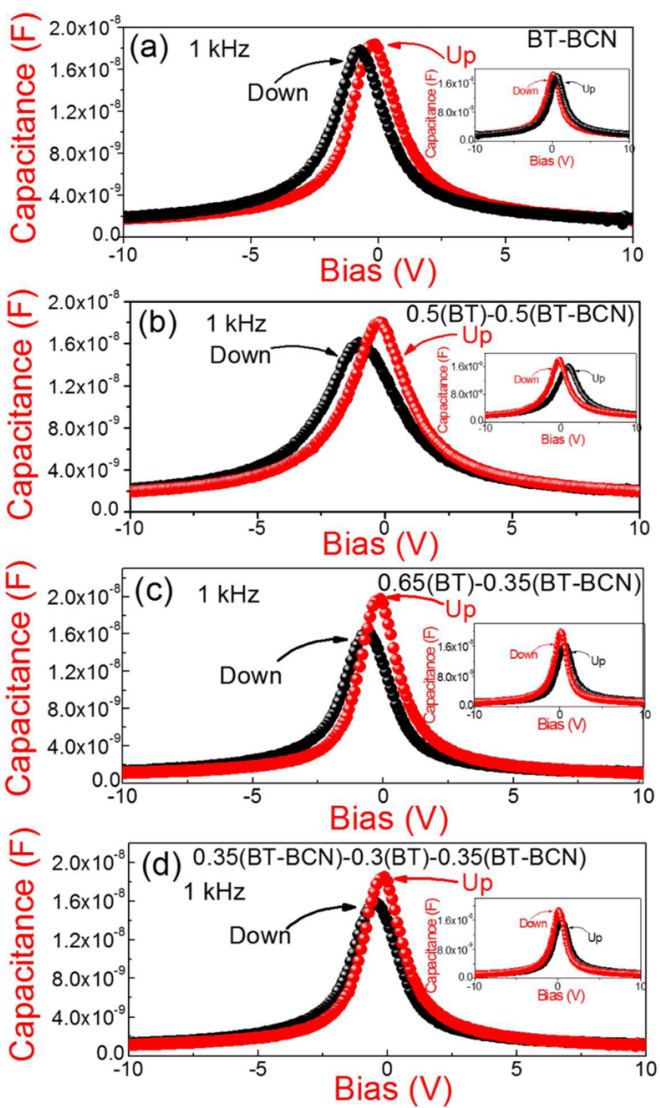

Figure 10. I Asymmetric capacitance-voltage plots due to built-in bias in functionally graded thin film heterostructure devices. Capacitance versus voltage plots for (a) $\mathrm{BT}-\mathrm{BCN}$ single layer, (b) 0.5(BT) $0.5(\mathrm{BT}-\mathrm{BCN})$ bilayer with equal thickness, (c) $0.35(\mathrm{BT})-0.65(\mathrm{BT}-$ $\mathrm{BCN}$ ) bilayer with unequal thickness, and (d) $0.35(\mathrm{BT}-\mathrm{BCN})-$ $0.30(\mathrm{BT})-0.35(\mathrm{BT}-\mathrm{BCN})$ trilayer functionally graded thin films. (Insets) Capacitance versus voltage plots measured under similar conditions but with bottom electrode as drive and top electrode as return.

$$
\begin{aligned}
& 2 \mathrm{BaO}+\mathrm{Nb}_{2} \mathrm{O}_{5} \\
& \rightleftharpoons 2 \mathrm{Ba}_{\mathrm{Ba}}^{\times}+2 \mathrm{Nb}_{\mathrm{Ti}}^{\bullet}+2 \mathrm{e}^{\prime}+6 \mathrm{O}_{\mathrm{O}}^{\times}+\frac{1}{2} \mathrm{O}_{2}(\mathrm{~g})
\end{aligned}
$$

where $\mathrm{Nb}_{\mathrm{Ti}}^{\bullet}$ and $\mathrm{e}^{\prime}$ are the singly charged dopant ions occupying the titanium sites and the electrons in the conduction band, respectively. The substitution of $\mathrm{Nb}^{5+}$ can also occur on the $\mathrm{Ti}^{4+}$ site leading to the formation of vacancy at the Ba site $\left(\mathrm{V}_{\mathrm{Ba}}^{\prime \prime}\right)$ :

$$
\mathrm{BaO}+\mathrm{Nb}_{2} \mathrm{O}_{5} \rightleftharpoons \mathrm{Ba}_{\mathrm{Ba}}^{\times}+2 \mathrm{Nb}_{\mathrm{Ti}}^{\bullet}+\mathrm{V}_{\mathrm{Ba}}^{\prime \prime}+6 \mathrm{O}_{\mathrm{O}}^{\times}
$$

However, the formation of Ba vacancies $\left(\mathrm{V}_{\mathrm{Ba}}^{\prime \prime}\right)$ has been considered energetically unfavorable. ${ }^{45}$ Therefore, possibility of the dominant presence of $\left(\mathrm{V}_{\mathrm{Ba}}^{\prime \prime}\right)$ can be ruled out. ${ }^{45}$ Furthermore, the negatively charged $\mathrm{Cu}_{\mathrm{Ti}}^{\prime \prime}$ can interact with the positively charged $\mathrm{V}_{\mathrm{O}}^{\bullet \bullet}$ leading to the formation of the defect complexes: ${ }^{46}$

$$
\mathrm{Cu}_{\mathrm{Ti}}^{\prime \prime}+\mathrm{V}_{\mathrm{O}}^{\bullet \bullet} \rightleftharpoons\left(\mathrm{Cu}_{\mathrm{Ti}}^{\prime \prime}-\mathrm{V}_{\mathrm{O}}^{\bullet \bullet}\right)^{\times}
$$

The $\left(\mathrm{Cu}_{\mathrm{Ti}_{\mathrm{i}}^{\prime \prime}}^{\prime \prime} \mathrm{V}_{\mathrm{O}}^{\bullet \bullet}\right)^{\times}$defect dipoles have been found to be oriented along the crystallographic $c$ axis. ${ }^{46}$ The schematic representation of the formation of defect dipoles is shown in Figure 11. We believe that the dynamics of such point defects, 


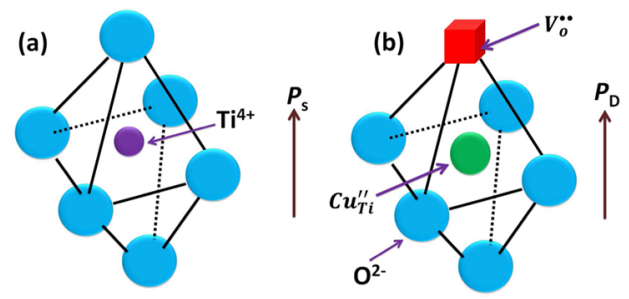

Figure 11. I Formation of oxygen vacancies and defect dipoles in BT$\mathrm{BCN}$ layer of functionally graded heterostructure devices. Schematic representation of the structural model (a) $\mathrm{T}^{4+}$ sites surrounded by oxygen octahedra in case of pure $\mathrm{BaTiO}_{3}(\mathrm{BT}),(\mathrm{b})$ the $\left(\mathrm{Cu}_{\mathrm{Ti}}^{\prime \prime}-\mathrm{V}_{\mathrm{O}}^{\bullet \bullet}\right)^{\times}$ defect dipole oriented along the crystallographic $c$ axis, creating axial center in $\mathrm{BT}-\mathrm{BCN}$ layer.

along with the defect complexes under the influence of the internal bias, can modulate the electrical transport behavior of a system. $^{47}$

The origin of an internal bias due to a polarization gradient in the functionally graded ferroelectric heterostructures can be further understood by invoking phenomenology. The total free energy for a coupled bilayer system ${ }^{11}$ can be given as

$$
\begin{aligned}
G_{\Sigma}= & (1-\alpha)\left[G_{1}\left(P_{1}\right)-E P_{1}-1 / 2 \xi E_{i 1} P_{1}\right]+\alpha\left[G_{2}\left(P_{2}\right)\right. \\
& \left.-E P_{2}-1 / 2 E_{i 2} P_{2}\right]+G_{S} / h+G_{e l}-\psi P_{1} P_{2}
\end{aligned}
$$

where $G_{1}$ and $G_{2}$ are free energy of layer 1 and 2 in its hightemperature $P E$ state, $P_{1}$ and $P_{2}$ are the polarizations of layers 1 and $2, G_{\mathrm{el}}$ is the elastic energy of the polarization-free misfit, and $\alpha$ is the relative volume fraction. The energy of interface $G_{S} / h$ ( $h$ is the thickness) can be neglected due to a very small correlation length of the ferroelectric ${ }^{48}$ in comparison to the total thickness of bilayer. The last term in above equation is the interlayer (interfacial) coupling and $\psi$ is the coupling coefficient (interfacial exchange interaction coefficient) given as ${ }^{11}$

$$
\psi=\alpha(1-\alpha) \xi / \varepsilon_{0}
$$

where $\xi$ is a measure of the free charge density with respect to the bound charge at the interlayer interface given as $\xi=1-\rho_{f}$ $/ \rho_{\mathrm{b}} ; \rho_{\mathrm{b}}$ is the bound charge density; and $\rho_{\mathrm{f}}$ is the free charge density. ${ }^{11}$ The two limiting values, $\xi=1$ and $\xi=0$ correspond to perfect insulating and semiconducting ferroelectric bilayers. ${ }^{11}$ The schematic of the total free energy $G_{\Sigma}$ for a homogeneous and functionally graded bilayer heterostructure is given in Figure 12. The difference in free energy $\Delta G$ between the components of functionally graded ferroelectric bilayer heterostructure was expected to result in the built-in potential:

$$
\Delta V=-\frac{x}{\Delta P} \Delta G
$$

where $\Delta P$ is the difference in polarization. ${ }^{11}$ The presence of internal field led to the skewed potential well, which consequently affected the switching characteristics of the functionally graded heterostructure under excitation of external bipolar signal. During hysteresis measurements under bipolar signal on functionally graded heterostructure thin film, ion rattles about their respective potential well in low field without any transition among arbitrarily occupied double wells. ${ }^{11}$ On increasing the magnitude of external signal, the ions in the deepest well are still expected to remain unaffected denying contribution to the hysteresis. ${ }^{11}$ However, at this stage, there could be switching in the shallowest double well pairs. This switching would not be symmetric as a result of higher

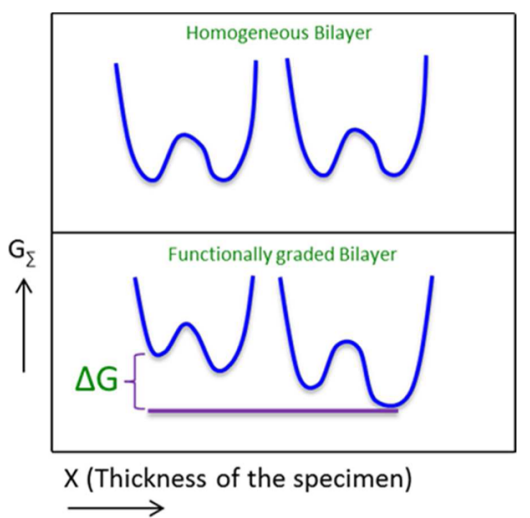

Figure 12. I Polarization gradient results in built-in internal bias. Schematic of the total free energy $\left(\mathrm{G}_{\Sigma}\right)$ diagram for a homogeneous and functionally graded bilayer heterostructure system. $\Delta G$ is the energy difference across the thickness of the functionally graded ferroelectric film.

probability of occupancy in lowest energy wells than that of the upper wells with lower energy. ${ }^{11}$ Consequently, a displacement of the hysteresis along the polarization axis was observed (Figure 5). The presence of net occupancy in the lowest potential wells was expected to shift the potential well (due to composition gradient), resulting in all wells becoming increasingly asymmetric. Furthermore, at higher magnitudes, the switching in deepest wells could result in an increasing tilting of potential wells with continued increase in the applied field. In the case of thin films, due to smaller thickness, the influence of grading could be expected to result in further increased skewness of the potential well leading to the modulation of electrical response (by modulating dynamics of charge carriers) with translated $P-E$ loop and asymmetric $I-V$ and $C-V$ characteristics.

3.5. Potential Applications of Functional Graded Architectures with Internal Electric Field. On the basis of the results presented above, these functionally graded heterostructures can find potential applications, as shown in Figure 13. The presence of an internal electric field and resulting polarization offset can be used in fabricating high density ferroelectric memory devices (Figure 13a). One may achieve different polarization switching states in forward and reverse bias both rendering higher number of switching states to store more information. Incorporation of the ferromagnetic layers along with the ferroelectric layers in the functional thin film heterostructure device, will provide an opportunity to control polarization switching behavior using magnetic field. ${ }^{49}$ Furthermore, in functionally graded multilayer heterostructures, the charge offset (polarization offset) can be used to fabricate high sensitivity infrared detector. ${ }^{11}$ The effective pyroelectric constant due to polarization offset can be given as $p_{\text {eff }}=(1 /$ $A)(\Delta Q / \Delta T)$, where $A$ is the area of the device, $(\Delta Q / \Delta T)$ is the change in the DC charge offset with temperature. ${ }^{11}$ Engineering of internal electric fields and electrostatic interactions in functionally graded ferroelectric heterostructures can also be used to achieve manifold improvement in the electrocaloric response. ${ }^{50}$ On the basis of the experimental results presented in Figure 8, another interesting application of these structures can be in memristor (resistive switching applications). The memristor-like current-voltage characteristics were an outcome of the interfacial coupling/internal bias, as discussed before. According to eq 6, the interfacial coupling 
(a) Presence of internal bias results in the polarization offset and higher number of memory states

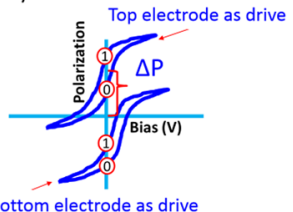

(b) Internal bias engineering can be used to achieve high performance resistive switching devices.

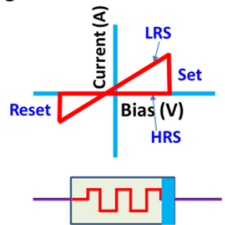

(c) Presence of internal electric field along with the depolarization field can be effectively used in photo-induced charge separation.

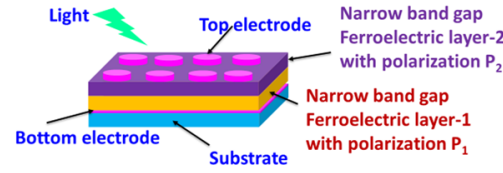

Figure 13. I Various proposed applications of functionally graded ferroelectric thin film heterostructures with built-in bias. (a) Highdensity ferroelectric memory, (b) memristor (resistive switching), and (c) solar energy harvesting through photovoltaic effect and water splitting $\left(\mathrm{H}_{2}\right.$ evolution).

coefficient $\psi$ in a bilayer graded heterostructure depends on $\xi$ (the free charge density with respect to the bound charge density) and $\alpha$ (the relative volume fraction in a bilayer). Depending upon the application, by selecting suitable dopant (donor or acceptor) and its concentration, and the volume fraction of the constituent layers, one can modulate the free charge density and interfacial coupling coefficient with minimum screening of the internal field. The engineering of the internal bias and fabrication of the highly epitaxial functionally graded heterostructure thin film, would be helpful in inducing high degree of resistive switching required for the memristor applications, as shown schematically in Figure 13b.

Furthermore, as demonstrated in the present work, the internal electric field in functionally graded ferroelectric heterostructure film was helpful in the photoinduced charge separation (Figure 9). The high efficiency and large current can be obtained by using such functional graded heterostructures composed of narrow band gap ferroelectric materials $(<3.0 \mathrm{eV})$, which will be helpful in effectively absorbing the visible light. ${ }^{51}$ The schematic of a solar energy harvesting device based on the functionally graded bilayer heterostructure is given in Figure 13c. The optimization of electrode configuration to efficiently collect charges will further result in enhanced photovoltaic effect. We believe that these structures can be used to efficiently harvest solar energy using photovoltaic effect and hydrogen evolution through water splitting. Moreover, oxide ferroelectric materials are generally very stable, which is vital for the extended operation of the devices under a harsh environment. It is worthwhile to mention that these functional device architectures can be integrated on silicon, as demonstrated in the present work. However, to fabricate epitaxial architectures on silicon, various buffer layers will have to be used rendering their scalability and cost-effective processing.

\section{CONCLUSIONS}

In summary, novel functionally graded interfaces in ferroelectric thin films were synthesized. The coupling at the interfaces was found to modulate the functional response in graded thin film heterostructures. These thin film structures exhibited shifting of the hysteresis loops on E-field and polarization axes depending on the direction of applied bias due to increased skewness of the potential wells. The thin film structures with graded interfaces further exhibited diode-like high rectification characteristics in current-voltage plots and asymmetric nature in current-voltage plots under forward and reverse bias both suggesting presence of strong internal bias. This novel modulated electrical behavior and built-in bias in these graded interfaces could find potential applications in thin film memory and other microelectronic devices and in modified form in solar energy harvesting devices.

\section{ASSOCIATED CONTENT}

\section{(S Supporting Information}

The Supporting Information is available free of charge on the ACS Publications website at DOI: 10.1021/acsami.5b06544.

$\mathrm{XRD}$-patterns recorded at room temperature for all the specimens, planar and cross-section SEM micrographs showing morphology of the various thin film architectures, the selected area electron diffraction (SAED), crater map after recording time-of-flight secondary-ion mass spectra, local piezoresponse hysteresis loops indicating polarization switching at nanoscale. (PDF)

\section{AUTHOR INFORMATION}

\section{Corresponding Authors}

*Tel.: (540) 231-0745. Fax: (540) 231-2903. E-mail: mauryad@vt.edu.

*E-mail: spriya@vt.edu.

\section{Author Contributions}

D.M. and S.P. conceived the idea and wrote the paper. D.M. designed experiments, synthesized targets for the pulsed laser deposition of thin films, and performed ferroelectric, electrical, and TEM characterizations. Y.Z. deposited thin films. B.C. performed photovoltaic measurements. M.-G.K. performed PFM measurements. P.N. performed cyclic $I-V$ measurements. M.K.H supervised cyclic $I-V$ measurements and provided suggestions on the manuscript. S.P. supervised the research. All authors discussed the results and commented on the manuscript.

Notes

The authors declare no competing financial interest.

\section{ACKNOWLEDGMENTS}

The authors gratefully acknowledge the financial support from Office of Basic Energy Sciences, Department of Energy (Y.Z. and S.P.; DE-FG02-07ER46405), Office of Naval Research through CEHMS (D.M. and M.-G.K.; N00014-14-1-0354) and National Science Foundation through INAMM program (B.C.; 26-1001-07 VTU).

\section{REFERENCES}

(1) Yang, H.; Luo, H. M.; Wang, H.; Usov, I. O.; Suvorova, N. A.; Jain, M.; Feldmann, D. M.; Dowden, P. C.; DePaula, R. F.; Jia, Q. X. Rectifying Current-Voltage Characteristics of $\mathrm{BiFeO}_{3} / \mathrm{Nb}$-doped $\mathrm{SrTiO}_{3}$ Heterojunction. Appl. Phys. Lett. 2008, 92 (10), 102113. 
(2) Miller, S. L.; McWhorter, P. J. Physics of the Ferroelectric Nonvolatile Memory Field Effect Transistor. J. Appl. Phys. 1992, 72 (12), 5999-6010.

(3) Ullmann, M.; Goebel, H.; Hoenigschmid, H.; Haneder, T. A BSIM3v3 and DFIM* Based Ferroelectric Field Effect Transistor Model. IEICE Trans. Electron. 2000, E83C (8), 1324-1330.

(4) Kim, K. H.; Han, J.-P.; Soon-Won, J.; Ma, T. P. Ferroelectric DRAM (FEDRAM) FET With Metal $/ \mathrm{SrBi}_{2} \mathrm{Ta}_{2} \mathrm{O}_{9} / \mathrm{SiN} / \mathrm{Si}$ Gate Structure. IEEE Electron Device Lett. 2002, 23 (2), 82-84.

(5) Wu, J.; Zhu, J.; Xiao, D.; Zhu, J. Double Hysteresis Loop in $\left(\mathrm{Pb}_{0.90} \mathrm{La}_{0.10}\right) \mathrm{Ti}_{0.975} \mathrm{O}_{3} / \mathrm{Pb}\left(\mathrm{Zr}_{0.20} \mathrm{Ti}_{0.80}\right) \mathrm{O}_{3}$ Bilayer Thin Films. Appl. Phys. Lett. 2007, 91 (21), 212905.

(6) Essaoudi, I.; Ainane, A.; Saber, M.; de Miguel, J. J. Hysteresis Loops of a Bilayer Superlattice. Phys. Status Solidi B 2007, 244 (9), 3398-3407.

(7) Wu, Y.-Z.; Yao, D.-L.; Li, Z.-Y. Hysteresis Loop of a Ferroelectric Bilayer With an Antiferroelectric Interfacial Coupling. J. Appl. Phys. 2002, 91 (3), 1482-1486

(8) Wu, S.; Luo, X.; Turner, S.; Peng, H.; Lin, W.; Ding, J.; David, A.; Wang, B.; Van Tendeloo, G.; Wang, J.; Wu, T. Nonvolatile Resistive Switching in $\mathrm{Pt} / \mathrm{LaAlO}_{3} / \mathrm{SrTiO}_{3}$ Heterostructures. Phys. Rev. X 2013, 3 (4), 041027.

(9) Li, L.; Salvador, P. A.; Rohrer, G. S. Photocatalysts With Internal Electric Fields. Nanoscale 2014, 6 (1), 24-42.

(10) Jiang, L.; Zhang, Y.; Qiu, Y.; Yi, Z. Improved Photocatalytic Activity by Utilizing the Internal Electric Field of Polar Semiconductors: A Case Study of Self-Assembled $\mathrm{NaNbO}_{3}$ Oriented Nanostructures. RSC Adv. 2014, 4 (7), 3165-3170.

(11) Mantese, J. V.; Alpay, S. P. Graded Ferroelectrics, Transpacitors and Transponents. Springer: New York, 2005.

(12) El-Naggar, M. Y.; Dayal, K.; Goodwin, D. G.; Bhattacharya, K. Graded Ferroelectric Capacitors With Robust Temperature Characteristics. J. Appl. Phys. 2006, 100 (11), 114115.

(13) Maurya, D.; Zhou, Y.; Wang, Y.; Yan, Y.; Li, J.; Viehland, D.; Priya, S. Giant Strain With Ultra-Low Hysteresis and High Temperature Stability in Grain Oriented Lead-Free $\mathrm{K}_{0.5} \mathrm{Bi}_{0.5} \mathrm{TiO}_{3}$ $\mathrm{BaTiO}_{3}-\mathrm{Na}_{0.5} \mathrm{Bi}_{0.5} \mathrm{TiO}_{3}$ Piezoelectric Materials. Sci. Rep. 2015, 5, 8595 .

(14) Maurya, D.; Zhou, Y.; Yan, Y.; Priya, S. Synthesis Mechanism of Grain-oriented Lead-Free Piezoelectric $\mathrm{Na}_{0.5} \mathrm{Bi}_{0.5} \mathrm{TiO}_{3}-\mathrm{BaTiO}_{3} \mathrm{Ce}-$ ramics with Giant Piezoelectric Response. J. Mater. Chem. C 2013, 1 (11), 2102-2111.

(15) Kundu, S.; Maurya, D.; Clavel, M.; Zhou, Y.; Halder, N. N.; Hudait, M. K.; Banerji, P.; Priya, S. Integration of Lead-Free Ferroelectric on $\mathrm{HfO}_{2} / \mathrm{Si}(100)$ for High Performance Non-Volatile Memory Applications. Sci. Rep. 2015, 5, 8494.

(16) Schubring, N. W.; Mantese, J. V.; Micheli, A. L.; Catalan, A. B.; Lopez, R. J. Charge Pumping and Pseudopyroelectric Effect in Active Ferroelectric Relaxor-type Films. Phys. Rev. Lett. 1992, 68 (11), 17781781

(17) Mantese, J. V.; Schubring, N. W.; Micheli, A. L.; Catalan, A. B.; Mohammed, M. S.; Naik, R.; Auner, G. W. Slater Model Applied to Polarization Graded Ferroelectrics. Appl. Phys. Lett. 1997, 71 (14), 2047-2049.

(18) Brazier, M.; McElfresh, M.; Mansour, S. Unconventional Hysteresis Behavior in Compositionally Graded $\mathrm{Pb}(\mathrm{Zr}, \mathrm{Ti}) \mathrm{O}_{3}$ Thin Films. Appl. Phys. Lett. 1998, 72 (9), 1121-1123.

(19) Bao, D.; Yao, X.; Zhang, L. Dielectric Enhancement and Ferroelectric Anomaly of Compositionally Graded ( $\mathrm{Pb}, \mathrm{Ca}) \mathrm{TiO}_{3}$ Thin Films Derived by a Modified Sol-Gel Technique. Appl. Phys. Lett. 2000, 76 (19), 2779-2781.

(20) Brazier, M.; McElfresh, M.; Mansour, S. Origin of Anomalous Polarization Offsets in Compositionally Graded $\mathrm{Pb}(\mathrm{Zr}, \mathrm{Ti}) \mathrm{O}_{3}$ Thin Films. Appl. Phys. Lett. 1999, 74 (2), 299-301.

(21) Misirlioglu, I. B.; Kesim, M. T.; Alpay, S. P. Strong Dependence of Dielectric Properties on Electrical Boundary Conditions and Interfaces in Ferroelectric Superlattices. Appl. Phys. Lett. 2014, 104 (2), 022906

(22) Maurya, D.; Kumar, A.; Petkov, V.; Mahaney, J. E.; Katiyar, R. S.; Priya, S. Local Structure and Piezoelectric Instability in Lead-Free $(1-\mathrm{x}) \mathrm{BaTiO}_{3}-\mathrm{xA}\left(\mathrm{Cu}_{1 / 3} \mathrm{Nb}_{2 / 3}\right) \mathrm{O}_{3}(\mathrm{~A}=\mathrm{Sr}, \mathrm{Ca}, \mathrm{Ba})$ Solid Solutions. RSC Adv. 2014, 4 (3), 1283-1292.

(23) Maurya, D.; Wongdamnern, N.; Yimnirun, R.; Priya, S. Dielectric and Ferroelectric Response of Compositionally Graded Bilayer and Trilayer Composites of $\mathrm{BaTiO}_{3}$ and $0.975 \mathrm{BaTiO}_{3}-$ $0.025 \mathrm{Ba}\left(\mathrm{Cu}_{1 / 3} \mathrm{Nb}_{2 / 3}\right) \mathrm{O}_{3}$. J. Appl. Phys. 2010, 108 (12), 124111.

(24) Wada, S.; Yako, K.; Kakemoto, H.; Tsurumi, T.; Kiguchi, T. Enhanced Piezoelectric Properties of Barium Titanate Single Crystals with Different Engineered-Domain Sizes. J. Appl. Phys. 2005, 98 (1), 014109.

(25) McGilly, L. J.; Yudin, P.; Feigl, L.; Tagantsev, A. K.; Setter, N. Controlling Domain Wall Motion in Ferroelectric Thin Films. Nat. Nanotechnol. 2015, 10 (2), 145-150.

(26) Maurya, D.; Ahn, C.-W.; Zhang, S.; Priya, S. High Dielectric Composition in the System Sn-Modified $(1-\mathrm{x}) \mathrm{BaTiO}_{3}-\mathrm{xBa}-$ $\left(\mathrm{Cu}_{1 / 3} \mathrm{Nb}_{2 / 3}\right) \mathrm{O}_{3}, \mathrm{x}=0.025$ for Multilayer Ceramic Capacitors. J. Am. Ceram. Soc. 2010, 93 (5), 1225-1228.

(27) Huang, H.; Zhong, X. L.; Xie, S. H.; Zhang, Y.; Wang, J. B.; Zhou, Y. C. Piezoresponse Force Microscopy Observation of Domain Switching in $\mathrm{Bi}_{3.15} \mathrm{Nd}_{0.85} \mathrm{Ti}_{3} \mathrm{O}_{12}$ Thin Film Prepared by Pulsed Laser Deposition. J. Appl. Phys. 2011, 110 (5), 054105.

(28) Okatan, M. B.; Roytburd, A. L.; Nagarajan, V.; Alpay, S. P. Electrical Domain Morphologies in Compositionally Graded Ferroelectric Films. J. Phys.: Condens. Matter 2012, 24 (2), 024215.

(29) Singh, S. K.; Ishiwara, H.; Sato, K.; Maruyama, K. Microstructure and Frequency Dependent Electrical Properties of Mnsubstituted BiFeO3 Thin Films. J. Appl. Phys. 2007, 102, 094109.

(30) Watanabe, Y. Energy Band Diagram of Ferroelectric Heterostructures and its Application to the Thermodynamic Feasibility of Ferroelectric FET. Solid State Ionics 1998, 108 (1-4), 59-65.

(31) Watanabe, Y. Electrical Transport Through $\mathrm{Pb}(\mathrm{Zr}, \mathrm{Ti}) \mathrm{O}_{3} p-n$ and $p-p$ Heterostructures Modulated by Bound Charges at a Ferroelectric Surface: Ferroelectric $p-n$ Diode. Phys. Rev. B: Condens. Matter Mater. Phys. 1999, 59 (17), 11257-11266.

(32) Choi, T.; Jiang, L.; Lee, S.; Egami, T.; Lee, H. N. High Rectification and Photovoltaic Effect in Oxide Nano-Junctions. New J. Phys. 2012, 14 (9), 093056.

(33) Fox, G. R.; Krupanidhi, S. B. Nonlinear Electrical Properties of Lead-Lanthanum-Titanate Thin Films Deposited by Multi-Ion-Beam Reactive Sputtering. J. Appl. Phys. 1993, 74 (3), 1949-1959.

(34) Giri, P. K.; Mohapatra, Y. N. Compensating Defect in Deep Buried Layers Produced by MeV Heavy Ions in n-Silicon. Appl. Phys. Lett. 1997, 71 (12), 1682-1684.

(35) Zhang, X. G.; Pantelides, S. T. Theory of Space Charge Limited Currents. Phys. Rev. Lett. 2012, 108 (26), 266602.

(36) Morrison, F. D.; Zubko, P.; Jung, D. J.; Scott, J. F.; Baxter, P.; Saad, M. M.; Bowman, R. M.; Gregg, J. M. High-Field Conduction in Barium Titanate. Appl. Phys. Lett. 2005, 86 (15), 152903.

(37) Rose, A. Space-Charge-Limited Currents in Solids. Phys. Rev. 1955, 97 (6), 1538-1544.

(38) Scott, J. F.; Araujo, C. A.; Melnick, B. M.; McMillan, L. D.; Zuleeg, R. Quantitative Measurement of Space-Charge Effects in Lead Zirconate-Titanate Memories. J. Appl. Phys. 1991, 70 (1), 382-388.

(39) Shuai, Y.; Ou, X.; Luo, W.; Mucklich, A.; Burger, D.; Zhou, S.; Wu, C.; Chen, Y.; Zhang, W.; Helm, M.; Mikolajick, T.; Schmidt, O. G.; Schmidt, H. Key Concepts Behind Forming-Free Resistive Switching Incorporated With Rectifying Transport Properties. Sci. Rep. 2013, 3, 2208.

(40) Yang, M.; Qin, N.; Ren, L. Z.; Wang, Y. J.; Yang, K. G.; Yu, F. M.; Zhou, W. Q.; Meng, M.; Wu, S. X.; Bao, D. H.; Li, S. W. Realizing a Family of Transition-Metal-Oxide Memristors Based on Volatile Resistive Switching at a Rectifying Metal/Oxide Interface. J. Phys. D: Appl. Phys. 2014, 47 (4), 045108.

(41) Park, S.-J.; Lee, J.-P.; Jang, J. S.; Rhu, H.; Yu, H.; You, B. Y.; Kim, C. S.; Kim, K. J.; Cho, Y. J.; Baik, S.; Lee, W. In situ Control of Oxygen Vacancies in $\mathrm{TiO}_{2}$ by Atomic Layer Deposition for Resistive Switching Devices. Nanotechnology 2013, 24 (29), 295202.

(42) Catlow, C. R. A. Defect Processes and Migration Mechanisms in Solid State Ionics. Mater. Sci. Eng., B 1992, 12 (4), 375-382. 
(43) Joshi, P. C.; Desu, S. B. Structural, Electrical, and Optical Studies on Rapid Thermally Processed Ferroelectric $\mathrm{BaTiO}_{3}$ Thin Films Prepared by Metallo-Organic Solution Deposition Technique. Thin Solid Films 1997, 300 (1-2), 289-294.

(44) Eichel, R.-A. Structural and Dynamic Properties of Oxygen Vacancies in Perovskite Oxides-Analysis of Defect Chemistry by Modern Multi-Frequency and Pulsed EPR Techniques. Phys. Chem. Chem. Phys. 2011, 13 (2), 368-384.

(45) Chan, H. M.; Harmer, M. R; Smyth, D. M. Compensating Defects in Highly Donor-Doped $\mathrm{BaTiO}_{3}$. J. Am. Ceram. Soc. 1986, 69 (6), 507-510.

(46) Eichel, R.-A.; Erhart, P.; Träskelin, P.; Albe, K.; Kungl, H.; Hoffmann, M. J. Defect-Dipole Formation in Copper-Doped $\mathrm{PbTiO}_{3}$ Ferroelectrics. Phys. Rev. Lett. 2008, 100 (9), 095504.

(47) Ren, X. Large Electric-Field-Induced Strain in Ferroelectric Crystals by Point-Defect-Mediated Reversible Domain Switching. Nat. Mater. 2004, 3 (2), 91-94.

(48) Strukov, B.; Levanyuk, A. Ferroelectric Phenomena in Crystals. Springer: New York, 1998.

(49) Valencia, S.; Crassous, A.; Bocher, L.; Garcia, V.; Moya, X.; Cherifi, R. O.; Deranlot, C.; Bouzehouane, K.; Fusil, S.; Zobelli, A.; Gloter, A.; Mathur, N. D.; Gaupp, A.; Abrudan, R.; Radu, F.; Barthélémy, A.; Bibes, M. Interface-Induced Room-Temperature Multiferroicity in $\mathrm{BaTiO}_{3}$. Nat. Mater. 2011, 10, 753-758.

(50) Kesim, M. T.; Zhang, J.; Alpay, S. P.; Martin, L. W. Enhanced Electrocaloric and Pyroelectric Response from Ferroelectric Multilayers. Appl. Phys. Lett. 2014, 105 (5), 052901.

(51) Nechache, R.; Harnagea, C.; Li, S.; Cardenas, L.; Huang, W.; Chakrabartty, J.; Rosei, F. Bandgap Tuning of Multiferroic Oxide Solar Cells. Nat. Photonics 2015, 9, 61-67. 